\title{
Perchlorethen-Quellendifferenzierung mittels Kohlenstoff-Chlor- Isotopenanalyse: Felduntersuchungen zur Beurteilung der Variabilität der Isotopensignatur
}

\author{
Alice Badin • Mario Schirmer • Christiane Wermeille • Daniel Hunkeler
}

Eingang des Beitrages: 13.2.2015 / Eingang des überarbeiteten Beitrages: 14.6.2015 / Online veröffentlicht: 9.11.2015

(C) Springer-Verlag Berlin Heidelberg 2015

\begin{abstract}
Zusammenfassung Bei der Untersuchung von belasteten Standorten stellt sich häufig die Frage, ob unterschiedliche Schadstoffquellen zu einer Grundwasserbelastung beitragen. Chlorierte Kohlenwasserstoffe (CKWs) von verschiedenen Produzenten können unterschiedliche Kohlenstoffund Chlor-Isotopensignaturen (C-Cl-Isotopensignaturen) aufweisen, was eine Differenzierung von CKW-Quellen ermöglicht. Dazu muss aber die Variabilität der Isotopensignatur bekannt sein. Da die ursprünglich verwendeten Lösungsmittel meist nicht mehr zugänglich sind, werden in dieser Studie die Perchlorethen (PCE) C-Cl-Isotopensignaturen an belasteten Standorten in der Schweiz untersucht. Zehn Standorte wurden ausgewählt, die verschiedene
\end{abstract}

Zusatzmaterial online Zusätzliche Information ist in der Onlineversion dieses Artikels (doi:10.1007/s00767-015-0301-0) enthalten.

A. Badin $(\bowtie) \cdot$ Prof. Dr. M. Schirmer · Prof. Dr. D. Hunkeler

Centre for Hydrogeology and Geothermics (CHYN),

University of Neuchâtel,

Rue Emile Argand 11, 2000 Neuchâtel, Schweiz

E-Mail: alice.badin@unine.ch

Prof. Dr. D. Hunkeler

E-Mail: daniel.hunkeler@unine.ch

Prof. Dr. M. Schirmer

Department of Water Resources and Drinking Water, Eawag,

Swiss Federal Institute of Aquatic Science and Technology,

Überlandstraße 133, P.O. Box 611, 8600 Dübendorf, Schweiz

E-Mail: mario.schirmer@eawag.ch

C. Wermeille

Federal Office for the Environment (BAFU),

Papiermühlestraße 172, 3063 Ittigen, Schweiz

E-Mail: christiane.wermeille@bafu.admin.ch
Branchen und Landesregionen abdecken. Eine Variabilität der C-Cl-Isotopensignaturen zwischen einigen Standorten bestätigt die Anwendbarkeit dieser Methode zur Quellendifferenzierung. Gewisse Standorte zeigen jedoch ähnliche Isotopensignaturen. Deswegen ist der Erfolg dieser Identifikationsmethode standortspezifisch. Außerdem ist die Variabilität geringer als publizierte Isotopensignaturen von nordamerikanischen Herstellern. Es hat sich außerdem bestätigt, dass der biologische Abbau von PCE durch reduktive Dechlorierung bei der Identifikation der Kontaminationsquellen berücksichtigt werden muss.

Perchloroethene source delineation using carbonchlorine isotopic analysis: field investigations of isotopic signature variability

Abstract When dealing with contaminated sites, identifying the source of contamination is critical for regulatory purposes. For chlorinated ethenes, previous studies have shown that dual carbon-chlorine $(\mathrm{C}-\mathrm{Cl})$ stable isotope analysis could be a key to address this issue as isotopic signatures vary between manufacturers and therefore, supposedly between sources. A successful application of this method relies on the assumption that different sources in the field will also show different signatures. Since the solvents used in the past are no longer available, this study aimed at investigating the extent of applicability of $\mathrm{C}-\mathrm{Cl}$ stable isotope measurements for source identification based on field investigations. Ten sites which covered all of Switzerland and various sectors employing perchloroethene (PCE) were chosen. Differences were observed between some sites, suggesting that this method could be successfully applied. Other sites showed very similar isotopic signatures, indicating that this method applicability is 
site-specific. Additionally, the isotopic signature variability between sites was less significant than between the values previously reported for solvents from various manufacturers from North America. It was also confirmed that PCE reductive dechlorination should be considered when applying $\mathrm{C}-\mathrm{Cl}$ isotope analysis for source identification.

Keywords Chlorinated ethenes · Isotopic signature · Source identification $\cdot$ Contaminated site

\section{Einleitung}

Chlorierte Kohlenwasserstoffe (CKWs) wurden zwischen 1940 und 1970 zur Metallentfettung in der Uhren- und Autoindustrie, als Reinigungsmittel in chemischen Reinigungen, als Lösungsmittel in der Chemieindustrie und zur Verwertung von Schlachthausabfällen verwendet. Aufgrund unsachgemäßer Entsorgung und Unfällen sind an vielen Standorten diese Stoffe in den Untergrund gelangt und belasten häufig das Grundwasser (Pankow \& Cherry 1996). Allein in der Schweiz sind im Kataster der belasteten Standorte ca. 12.300 CKW-belastete Standorte (Betriebsstandorte und Deponien) eingetragen.

Wenn ein Standort saniert werden muss, ist es wichtig, den Verursacher zu identifizieren, da er für die Sanierungskosten verantwortlich ist. Da die Bestimmung der Kontaminationsquelle durch klassische hydrogeologische und hydrochemische Studien nicht immer erreicht werden kann, ist das Interesse für alternative Methoden groß.

Vorherige Studien haben gezeigt, dass sich die Kohlenstoff- und Chlor-Isotopensignatur (C-Cl-Isotopensignatur) von reinem Perchlorethen (PCE) zwischen einzelnen Herstellern markant unterscheiden kann (van Warmerdam 1995; Jendrzejewski et al. 2001; McHugh et al. 2011). Deshalb bietet sich die Analyse des stoffspezifischen Isotopenverhältnisses als nützliches Werkzeug zur Unterscheidung verschiedener Schadstoffquellen an, dessen Anwendung bereits in einigen Studien erfolgreich bestätigt wurde (McHugh et al. 2011; Lojkasek-Lima et al. 2012; Kaown et al. 2014; Palau et al. 2014). Richtlinien für die Anwendung der Methode wurden zudem publiziert (Hunkeler et al. 2008; Eisenmann \& Fischer 2010). Bisherige Anwendungen beschränkten sich meist auf Kohlenstoffisotope (Hunkeler et al. 2004; Eberts et al. 2008; Blessing et al. 2009; Amaral et al. 2011; Nijenhuis et al. 2013), während die kombinierte $\mathrm{C}$-Cl-Isotopenanalyse erst in geringem Maße angewandt wurde (McHugh et al. 2011; Lojkasek-Lima et al. 2012; Kaown et al. 2014; Palau et al. 2014). Dies ist damit zu erklären, dass sich die Bestimmung des Chlor-Isotopenverhältnisses von Feldproben erst in den letzten Jahren etabliert hat und zertifizierte Referenzstandards für organische Verbindungen noch fehlen (Sakaguchi-Söder et al. 2007; Aep- pli et al. 2010; Bernstein et al. 2011; Hitzfeld et al. 2011; Jin et al. 2011). Damit die Anwendung von C-Cl-Isotopenverhältnismessungen zur Quellenidentifizierung routinemäßig angewandt werden kann, sollte man aber sicherstellen, dass ein Unterschied der C-Cl-Isotopensignaturen auch im Feld existiert. Obwohl die Variabilität des Isotopenverhältnisses zwischen Herstellern vor allem in Nordamerika bereits untersucht wurde, mangelt es jedoch an Erfahrungen hinsichtlich der Variabilität bei Felduntersuchungen in Europa. Zudem ist es unklar, ob die in der Literatur dokumentierte Variabilität auch für Europa und insbesondere die Schweiz repräsentativ ist. Außerdem existiert keine Angabe zur Herkunft des in der Schweiz verwandten PCE und dessen Isotopensignatur, im Vergleich zu den USA (Doherty 2000; Moran et al. 2007). Deshalb untersuchen wir die Variabilität des PCE-C-Cl-Isotopenverhältnisses an belasteten Standorten im Feld. Grundwasserproben wurden im Quellenbereich von zehn PCE-belasteten Standorten in der Schweiz entnommen, um die Variabilität des Isotopenverhältnisses zu untersuchen. Die Standorte repräsentieren dabei unterschiedliche Verwendungszwecke von PCE und befinden sich in verschiedenen Regionen der Schweiz. Die gemessenen Isotopensignaturen wurden untereinander und mit den publizierten C-Cl-Isotopenverhältnissen von PCE der verschiedenen Hersteller verglichen.

\section{Methoden und Materialien}

Methode zur Standortauswahl

Bei der Auswahl der Standorte wurden mehrere Kriterien verwendet:

1. Der biologischer Abbau sollte vernachlässigbar sein, da dieser zu einer Verschiebung der Isotopensignatur führt (Hunkeler et al. 1999; Lollar et al. 1999; Bloom et al. 2000). Standorte sollten entsprechend oxische Bedingungen und/oder nur geringe Konzentrationen an Abbauprodukten aufweisen.

2. Mehrere Probennahmestellen sollten im Bereich der Schadstoffquelle verfügbar sein.

3. Die Standorte sollten verschiedene Branchen und Landesregionen abdecken.

4. Der Eigentümer muss der Publikation der Daten zustimmen.

Aufgrund dieser Kriterien konnten zehn geeignete Standorte identifiziert werden. Die Standorte repräsentieren die drei typischen CKW-Nutzungsbereiche, d. h. chemische Reinigung (7), Metallentfettung (3) und Verarbeitung von Schlachthausabfällen (1). Die Hauptcharakteristiken der Standorte sind in der Tabelle 1 zusammengefasst. 
Tab. 1 Zusammenfassung der Geologie, Hydrogeologie, Geochemie und Vorgeschichte der zehn analysierten Standorte

\begin{tabular}{|c|c|c|c|c|c|c|c|}
\hline Standort & $\begin{array}{l}\text { Nutzungsbe- } \\
\text { reich }\end{array}$ & $\begin{array}{l}\text { Standortvorge- } \\
\text { schichte }\end{array}$ & $\begin{array}{l}\text { Fahnenbe- } \\
\text { schreibung }\end{array}$ & Geologie und Hydrogeologie & $n$ & Konzentrationsbereiche & $\begin{array}{l}\text { Redoxbe- } \\
\text { dingungen }\end{array}$ \\
\hline SA & $\begin{array}{l}\text { CR/Maschinen- } \\
\text { entfettung }\end{array}$ & $\begin{array}{l}\text { 1959-1990; } \\
10001 \text { Tank; } \\
\text { CKW-Phase } \\
\text { erwartet }\end{array}$ & $\begin{array}{l}\mathrm{L}:>80 \mathrm{~m} \\
\mathrm{~B}: \text { unb. } \\
\mathrm{T}: 40 \mathrm{~m}\end{array}$ & $\begin{array}{l}\text { Sand- und Kies-Aquifer mit } \\
\text { Tonschichten; GW-Spiegel: } \\
5 \text { m; Mächtigkeit des Aqui- } \\
\text { fers: } 34 \mathrm{~m}\end{array}$ & 3 & $\begin{array}{l}\text { PCE: } 1000-5900 \mu \mathrm{g}^{-1} \\
\text { TCE: }<1 \%(\mathrm{PCE}) \\
\text { cDCE: }<1 \%(\mathrm{PCE})\end{array}$ & $\begin{array}{l}\text { Oxidierend } \\
\text { bis mangan- } \\
\text { reduzierend }\end{array}$ \\
\hline SB & CR & 1968-1983 & $\begin{array}{l}\mathrm{L}:>70 \mathrm{~m} \\
\mathrm{~B}: u n b . \\
\mathrm{T}: \text { unb. }\end{array}$ & $\begin{array}{l}\text { Sand- und Kies-Aquifer mit } \\
\text { undurchlässigen Schichten; } \\
\text { GW-Spiegel: } 5 \mathrm{~m} \text {; Mächtig- } \\
\text { keit des Aquifers: } 4 \mathrm{~m}\end{array}$ & 5 & $\begin{array}{l}\text { PCE: } 100-6400 \mu \mathrm{g}^{-1} \\
\text { TCE: }<5 \mu \mathrm{g} \mathrm{l}^{-1} \\
\text { cDCE: }<5 \mu \mathrm{g} \mathrm{l}^{-1}\end{array}$ & Oxidierend \\
\hline $\mathrm{SC}$ & CR & $\begin{array}{l}\text { 1896-2006; Un- } \\
\text { fälle } 1989 \text { und } \\
1990\end{array}$ & $\begin{array}{l}\mathrm{L}: 240 \mathrm{~m} \\
\mathrm{~B}: 140 \mathrm{~m} \\
\mathrm{~T}:<4 \mathrm{~m}\end{array}$ & $\begin{array}{l}\text { Silt-, Sand- und Kies-Aqui- } \\
\text { fer; GW-Spiegel: } 2,5-3,5 \mathrm{~m} \text {; } \\
\text { Mächtigkeit des Aquifers: } \\
0,5-1,5 \mathrm{~m}\end{array}$ & 2 & $\begin{array}{l}\text { PCE: } 890-1700 \mu \mathrm{g} \mathrm{l}^{-1} \\
\text { TCE: }<5 \mu \mathrm{g} \mathrm{l}^{-1} \\
\text { cDCE: }<5 \mu \mathrm{g} \mathrm{l}^{-1}\end{array}$ & Oxidierend \\
\hline SD & Metallentfettung & $u n b$. & unb. & $\begin{array}{l}\text { Silt-Sand und Kies-Aquifer; } \\
\text { GW-Spiegel: } 2 \mathrm{~m} \text {; Mächtig- } \\
\text { keit des Aquifers: } 6,5 \mathrm{~m}\end{array}$ & 4 & $\begin{array}{l}\text { PCE: } 80-210 \mu \mathrm{g}^{-1} \\
\text { TCE: }<5 \mu \mathrm{g} \mathrm{l}^{-1} \\
\text { cDCE: }<5 \mu \mathrm{g} \mathrm{l}^{-1}\end{array}$ & Oxidierend \\
\hline SE & Metallentfettung & $\begin{array}{l}1955-1995 ; \\
\text { benutzte Menge: } \\
\text { mehrere t/a }\end{array}$ & $\begin{array}{l}\mathrm{L}:>160 \mathrm{~m} \\
\mathrm{~B}:>60 \mathrm{~m} \\
\mathrm{~T}: \text { unb. }\end{array}$ & $\begin{array}{l}\text { Sand- und Kies-Aquifer; } \\
\text { GW-Spiegel: 7-8 m; Mäch- } \\
\text { tigkeit des Aquifers: 4-5 m }\end{array}$ & 4 & $\begin{array}{l}\text { PCE: } 380-570 \\
\text { TCE }:<5 \\
\text { cDCE }:<5\end{array}$ & Oxidierend \\
\hline SF & $\begin{array}{l}\text { Tierkörperbe- } \\
\text { seitigung }\end{array}$ & $\begin{array}{l}15 \text { Jahre lange } \\
\text { Aktivität; abge- } \\
\text { schätzte verlorene } \\
\text { Menge: } 3600 \mathrm{~kg}\end{array}$ & $\begin{array}{l}\text { L: } 750 \mathrm{~m} \\
\text { B: } 100 \mathrm{~m} \\
\text { T: unb. }\end{array}$ & $\begin{array}{l}\text { Kies-Aquifer; GW-Spiegel: } \\
\text { 2-3 m; Mächtigkeit des } \\
\text { Aquifers: } 2-4 \mathrm{~m}\end{array}$ & 2 & $\begin{array}{l}\text { PCE: } 630-650 \mu \mathrm{g}^{-1} \\
\text { TCE: }<5 \mu \mathrm{g}^{-1} \\
\text { cDCE: }<5 \mu \mathrm{g} \mathrm{l}^{-1}\end{array}$ & $\begin{array}{l}\text { Oxidierend } \\
\text { bis mangan- } \\
\text { reduzierend }\end{array}$ \\
\hline SG & $\mathrm{CR}$ & 1974-1990 & $u n b$. & $\begin{array}{l}\text { Kies- und Sand-Aquifer mit } \\
\text { Silt- und Mergel-Schichten; } \\
\text { GW-Spiegel: } 4-5 \mathrm{~m} \text {; Mäch- } \\
\text { tigkeit des Aquifers: } 3 \mathrm{~m}\end{array}$ & 3 & $\begin{array}{l}\text { PCE: } 890-132.000 \mu \mathrm{g}^{-1} \\
\text { TCE: }<4 \% \text { (PCE) } \\
\text { cDCE: }<1 \% \text { (PCE) }\end{array}$ & Oxidierend \\
\hline SH & CR & $1966-1988$ & unb. & $\begin{array}{l}\text { Silt-, Sand- und Kies-Aquifer } \\
\text { mit Silt-Schichten; GW- } \\
\text { Spiegel: } 6 \mathrm{~m} \text {; Mächtigkeit } \\
\text { des Aquifers: } 1 \mathrm{~m}\end{array}$ & 2 & $\begin{array}{l}\text { PCE: } 70-600 \mu \mathrm{g} \mathrm{l}^{-1} \\
\text { TCE: }<5 \mu \mathrm{g} \mathrm{l}^{-1} \\
\text { cDCE: }<5 \mu \mathrm{g} \mathrm{l}^{-1}\end{array}$ & Oxidierend \\
\hline SI & CR & $u n b$. & $\begin{array}{l}\mathrm{L}: 100 \mathrm{~m} \\
\mathrm{~B}: 35 \mathrm{~m} \\
\mathrm{~T}: \text { unb. }\end{array}$ & $\begin{array}{l}\text { Silt-, Sand- und Kies-Aquifer } \\
\text { mit einem Hohlraum unter- } \\
\text { halb des Quellenbereich; } \\
\text { GW-Spiegel: } 3 \text { m; Mächtig- } \\
\text { keit des Aquifers: } 1-4 \mathrm{~m}\end{array}$ & 4 & $\begin{array}{l}\text { PCE: } 37.000-150.000 \mu \mathrm{g}^{-1} \\
\text { TCE: }<1 \% \text { (PCE) } \\
\text { cDCE: }<1 \% \text { (PCE) }\end{array}$ & Oxidierend \\
\hline
\end{tabular}

$n$ Zahl der beprobten Bohrungen, $L$ Länge, $B$ Breite, $T$ Tiefe, unb. unbekannt, $C R$ chemische Reinigung, $G W$ Grundwasser

Grundwasserprobennahme

Die Probennahmen wurden zwischen März und August 2012 durchgeführt und haben jeweils einen Tag pro Standort gedauert. Je nach Anzahl der Messstellen in Quellennähe und deren PCE-Konzentration, wurden pro Standort zwei bis fünf Messstellen geprobt. Es wurde vor der Probennahme Grundwasser gepumpt bis die Feldparameter stabil waren ( $\mathrm{pH}$, elektrische Leitfähigkeit, Sauerstoffkonzentration). Normalerweise wurde eine Tauchpumpe eingesetzt, außer bei den tiefenorientiert ausgebauten Bohrungen, wo eine Fußventil- oder eine Peristaltikpumpe verwendet wurde. Um CKW-Sorption während der Probennahme zu vermeiden, wurde ein Schlauch aus Teflon benutzt. Die Grundwasserproben für Isotopen- und Konzentrationsanalysen wurden ohne Gasphase in ein $40 \mathrm{ml}$ Glasvial mit PTFE-beschichtetem Silikonseptum und Schraubdeckel abgefüllt. Zudem wurde Salpetersäure (10\%) zu den Grundwasserproben zugefügt, um einen pH-Wert von $2 \mathrm{zu}$ erreichen und somit einen Abbau während der Lagerung zu vermeiden. Die Proben wurden in Kühlboxen mit Eis transportiert und in einem Kühlraum bei $4{ }^{\circ} \mathrm{C}$ gelagert. Der pH-Wert, die elektrische Leitfähigkeit und die Sauerstoffkonzentration wurden im Feld gemessen (HQ40d-Sonde, Hach). Wenn die Sauerstoffkonzentration tiefer als $1 \mathrm{mg} \cdot 1^{-1}$ war, wurden zudem $\mathrm{Mn}^{2+}$ - und $\mathrm{Fe}^{2+}$ Konzentrationen mit einem tragbaren Colorimeter (DR 890, Hach) gemessen, um den Grad der reduzierenden Bedingungen abzuschätzen.

Analytische Methoden

CKW-Konzentrationen wurden mit einem Trace ${ }^{\mathrm{TM}} \mathrm{GC}$ Ultra Gas-Chromatograph (Thermo-Finnigan ${ }^{\mathrm{TM}}$ ) gemessen, welcher mit einem DSQ II Quadrupol-Massenspektrometer (GC-qMS, Thermo-Finnigan ${ }^{\mathrm{TM}}$ ) gekoppelt war. Für die Analyse wurden $500 \mu \mathrm{l}$ vom Headspace eines halbgefüllten $20 \mathrm{ml}$ Glasvials mithilfe eines CombiPal-Autosampler (CTC Analytics) in den Gaschromatographen injiziert. 
Das Isotopenverhältnis von Element $\mathrm{E}$ wurde in der delta-Notation im Verhältnis zu einem internationalen Standard (Vienna Pee Dee Belemnite $=$ VPDB für C oder Standard Mean Ocean Chloride $=\mathrm{SMOC}$ für $\mathrm{Cl}$ ) in \%o angegeben:

$$
\delta^{i} E=\frac{R_{P}}{R_{s t d}}-1
$$

wobei $\mathrm{R}_{\mathrm{p}}$ und $\mathrm{R}_{\text {std }}$ die Isotopenverhältnisse von Element $\mathrm{E}$ (d. h. hier $\mathrm{C}\left({ }^{13} \mathrm{C} /{ }^{12} \mathrm{C}\right)$ oder $\left.\mathrm{Cl}\left({ }^{37} \mathrm{Cl} /{ }^{35} \mathrm{Cl}\right)\right)$ der Probe beziehungsweise des internationalen Standards bezeichnen und $\mathrm{i}$ relativ zum schweren Isotop ist.

$\mathrm{C}$-und Cl-Isotopenanalysen wurden wie bereits beschrieben durchgeführt (Badin et al. 2014). Kohlenstoff-Isotopenverhältnisse wurden mit einem ,isotope ratio mass spectrometer" (IRMS, Isoprime ${ }^{\mathrm{TM}} 100$ ) gemessen, nachdem die Wasserprobe durch eine Probenbehandlungskette zur Messung vorbereitet wurde. Dies geschah in den folgenden Schritten: Die CKWs in den Wasserproben wurden mittels eines ,purge and trap“ Systems (Tekmar Stratum PTC) konzentriert, desorbiert und in einer Kühlfalle mit Flüssigstickstoff aufgefangen, bevor sie mittels eines Gaschromatographen (Agilent 7890A; DB-VRX GC-Säule, $60 \mathrm{~m}, 0,25 \mathrm{~mm}, 1,4 \mu \mathrm{m})$ aufgetrennt wurden. Die Ofentemperatur wurde für $6 \mathrm{~min}$ auf $40^{\circ} \mathrm{C}$ gehalten, gefolgt von einer Temperaturerhöhung von $10^{\circ} \mathrm{C} / \mathrm{min}$ bis $130^{\circ} \mathrm{C}$. Diese Temperatur wurde für 0,1 min gehalten. Schließlich wurde eine weitere Temperaturerhöhung von $20^{\circ} \mathrm{C} / \mathrm{min}$ bis $220^{\circ} \mathrm{C}$ durchgeführt. Diese wurde dann 1 min gehalten. Anschließend wurden die CKWs in einem Hochtemperaturofen (Thermo-Finnigan ${ }^{\mathrm{TM}}$ ) $\mathrm{zu} \mathrm{CO}_{2}$ verbrannt und danach zum Massenspektrometer transportiert, wo die C-Isotopenverhältnisse $\left({ }^{13} \mathrm{C} /{ }^{12} \mathrm{C}\right)$ gemessen wurden. Jeweils nach 10 Proben wurde ein Labor-Referenz-PCE injiziert, welches mittels Elementar-Analysator - IRMS eingemessen wurde. Die Standardabweichung des Laborreferenz-PCEs wurde als Ma $\beta$ für die Reproduzierbarkeit der Methode und zur Berechnung der Standardabweichung Typ A gemäß der ISO-Richtlinie (BIPM 1993) verwendet. Aufgrund der guten Auftrennung des PCEs und der stabilen Basislinie kann die Standardabweichung für das Referenz-PCE auch für die Proben als repräsentativ erachtet werden. Es wurde eine Standardabweichung von $0,2 \%$ o $(n=15)$ erhalten. Da jede Probe zweimal gemessen und der Mittelwert verwendet wurde, ergibt sich eine Standardabweichung für die Proben von $\sigma=0,2 / \sqrt{2}$.

Cl-Isotopenverhältnisse wurden mittels eines Gaschromatographen (Agilent 7890A) gekoppelt mit einem Quadrupol-Massenspektrometer (Agilent 5975C) gemessen. Die Molekülionen wurden basierend auf der in Aeppli et al. (2010) beschriebenen Methode analysiert. Die Rohdaten wurden mittels einer Zweipunktkalibrierung ausgewertet, wie von Bernstein et al. (2011) empfohlen wurde. Die dafür benutzten Standards wurden mittels der Holt-Methode (Holt et al. 1997) an der Universität von Waterloo charakterisiert $\left(\delta^{37} \mathrm{Cl}_{\mathrm{EIL} 1}=+0,3 \%\right.$ o und $\left.\delta^{37} \mathrm{Cl}_{\mathrm{EIL} 2}=-2,5 \%\right)$. Der Headspace jeder Probe wurde zehnmal gemessen, und die Standardabweichung des Mittelwerts wurde für jede Probe einzeln berechnet.

\section{Resultate und Diskussion}

Variabilität der Isotopensignatur innerhalb der Standorte

Die Isotopenverhältnisse aller Messstellen befinden sich in einem Bereich zwischen $-0,7$ und $0,8 \%$ für $\mathrm{Cl}$ und zwischen $-26,8$ und $-23,7 \%$ für $\mathrm{C}$ und unterscheiden sich zwischen den Messstellen an jedem Standort außer an den Standorten SC und SF (Abb. 1 und Tab. S 1, Ergänzende Informationen). Der maximale Unterschied zwischen dem Minimum und Maximum der C- und Cl-Isotopenverhältnisse beträgt $1,3 \%$ für $\mathrm{C}$ (Standort $\mathrm{SE}$ ) beziehungsweise $0,6 \%$ für $\mathrm{Cl}$ (Standorte SB, SE und SG) (Tab. 2). In den beiden vorher publizierten Studien, welche die reduktive PCE-Dechlorierung basierend auf C- und Cl-Isotopenverhältnissen im Feld untersucht haben (Wiegert et al. 2012; Badin et al. 2014), ist die Verschiebung von mindestens einem Element $(\mathrm{C}$ oder $\mathrm{Cl})$ größer als jene der zehn hier untersuchten Standorte (Tab. 2). Zudem zeigt das C vs. $\mathrm{Cl}$ Isotopendiagramm in acht von zehn Standorten kein lineares Verhältnis zwischen C- und Cl-Isotopen. Ein lineares Verhältnis wäre typisch für reduktive Dechlorierung. Die geringe standortspezifische Variabilität der Isotopensignatur unserer Studie ist deshalb eher auf verschiedene physikalische Prozesse (z. B. Diffusion, Sorption, Übertritt in ungesättigte Zone) oder auf verschiedene Schadstoffeinträge mit unterschiedlichen Primärisotopenverhältnissen zurückzuführen. Obwohl der Einfluss dieser Prozesse auf das Isotopenverhältnis als gering im Vergleich zum Abbauprozess einzustufen ist (Slater et al. 1999; Jeannottat \& Hunkeler 2012; Jeannottat \& Hunkeler 2013) könnten sie dennoch für die kleine standortspezifische Variabilität der Isotopensignatur verantwortlich sein. Obwohl Standorte ausgewählt wurden, die keine reduktive Dechlorierung zeigen, scheint es, als ob eine reduktive Dechlorierung trotzdem in zwei von zehn untersuchten Standorten stattfindet. Dies ist an den Standorten SA und SB ersichtlich, wo eine abbauprozessabhängige Korrelation zwischen den C-Cl-Isotopenverhältnissen entlang der Fließrichtung zu beobachten ist (Abb. S 1 und S 2, ergänzende Informationen). Deren Variabilität ist jedoch geringer als die bei den vorher untersuchten Standorten, die reduktive Dechlorierung aufzeigten (Tab. 2) (Wiegert et al. 2012; Badin et al. 2014). 
Abb. 1 C-Cl-Isotopenverhältnis für jede Messstelle von jedem untersuchten Standort (Standorte SA bis SJ) und C-Cl-Isotopenverhältnis verbunden mit PCE-reduktiver Dechlorierung, die an einem Standort stattfindet, hier jeweils vorher untersuchte Standorte $\mathrm{X}$ und $\mathrm{Y}$ (nach Badin et al. 2014), und Transekte 1 und 2 (jeweils T1 und T2) von einem Standort (nach Wiegert et al. 2012). Die gestrichelten Linien entsprechen der reduktiven Dechlorierung, die in vorherigen Studien aufgezeigt wurde

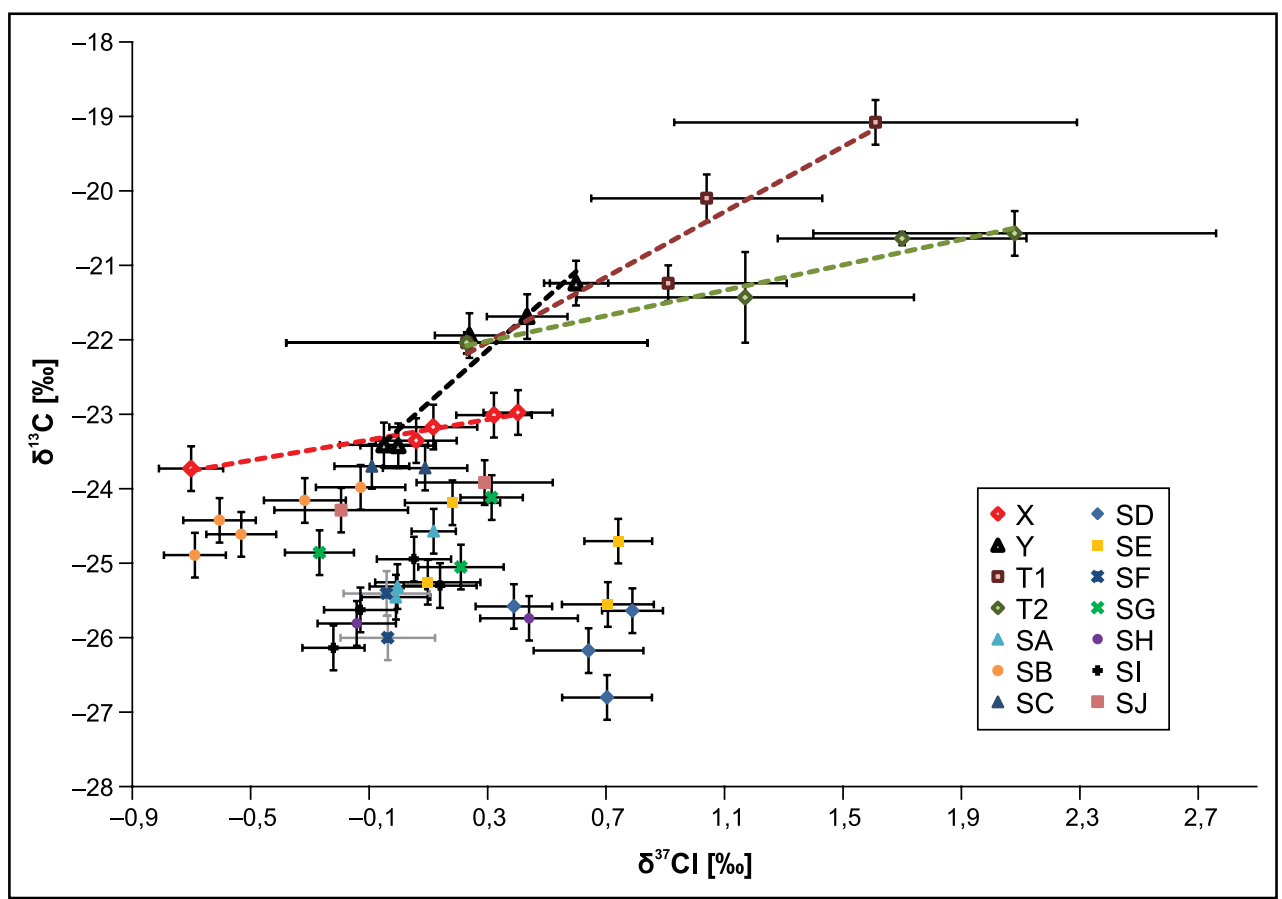

Variabilität der Isotopensignatur zwischen den Standorten

Der Mittelwert der Isotopenverhältnisse von allen Messstellen eines Standorts wurde als repräsentative Isotopensignatur verwendet (Tab. 2; Abb. 2 und 3), da physikalische Prozesse zu kleinen Abweichungen in beiden Richtungen führen können. Die Standartabweichung entspricht der Standartabweichung der Messwerte aller Messstellen eines Standortes oder der analytischen Messunsicherheit, falls die letztere größer ist.

Die C-Cl-Variabilität der Isotopensignatur zwischen den zehn untersuchten Schweizer Standorten ist weniger markant als die bisher veröffentlichten Variationen von PCESignaturen zwischen einzelnen CKW-Herstellern (van Warmerdam 1995; Jendrzejewski et al. 2001; McHugh et al. 2011), und sie ist unabhängig vom Industriebereich oder der geographischen Lage (Abb. 2 und Tab. 1). CKWs wurden an den Standorten SA, SD und SE zur Metallentfettung benutzt, während sie zur Verwertung von Schlachtausabfällen am Standort SF beziehungsweise zur chemischen Reinigung an den Standorten SA, SB, SC, SG, SH, SI und SJ verwendet wurden. Es kann kein Cluster der Isotopensignatur identifiziert werden, der spezifisch zu einem dieser drei CKW-Nutzungsbereiche passen würde. Die Standorte SA $\left(-25,1 \%_{0} \pm 0,5 ; 0,1 \%{ }_{0} \pm 0,1\right)^{1}$ und SE $(-24,9 \% \pm 0,6$; $0,4 \% \pm 0,3)$ sind ca. $250 \mathrm{~km}$ voneinander entfernt und zeigen trotzdem ähnliche Isotopensignaturen. Ebenso zeigen die Standorte SA $(-25,1 \% \pm 0,5 ; 0,1 \% \pm 0,1)$ und SC

\footnotetext{
${ }^{1}$ Zur Vereinfachung des Leseflusses sind die Isotopensignaturen im
} Text als $\left(\delta^{13} \mathrm{C}(\%) ; \delta^{37} \mathrm{Cl}(\%)\right)$ angegeben. $\left(-23,7 \%_{0} \pm 0,1 ; 0,0 \% \pm 0,1\right)$, die ca. $300 \mathrm{~km}$ voneinander entfernt liegen, eine ähnliche Isotopensignatur, während die Standorte SD $\left(-26,0 \%_{0} \pm 0,6 ; 0,6 \% \pm 0,2\right)$ und SC $\left(-23,7 \% \%_{0} \pm 0,1 ; 0,0 \% \pm 0,1\right)$, die nur $80 \mathrm{~km}$ voneinander entfernt sind, eine unterschiedliche Isotopensignatur aufweisen. Trotz der geringen Variabilität, weisen gewisse Standorte eine unterschiedliche Signatur auf (z. B. Standorte SB $\left(-24,4 \%{ }_{0} \pm 0,4 ;-0,5 \%{ }_{0} \pm 0,2\right)$ und SD $\left(-26,0 \%{ }_{0} \pm 0,6\right.$; $0,6 \% \pm 0,2)$ ), während andere nicht unterscheidbar sind (z. B. Standorte SF $(-25,7 \% \pm \pm 0,3 ; 0,0 \% \pm 0,2)$ und SI $\left(-25,5 \%_{0} \pm 0,5 ; 0,0 \% \pm 0,2\right)$ ) (Abb. 3; Tab. 2). Obwohl die verschiedenen $\mathrm{CKW}$-Anwendungsgebiete gut repräsentiert sind und die Standorte weit voneinander entfernt liegen, muss man berücksichtigen, dass damit erst zehn von ca. 12.300 CKW-belasteten Standorten untersucht wurden. Es kann deswegen nicht ausgeschlossen werden, dass die Variabilität der Isotopensignatur zwischen anderen Schweizer Standorten größer ist.

\section{Auswirkung der Resultate auf der Praxis}

Im Allgemeinen kann die C-Cl-Isotopenanalyse eingesetzt werden, um verschiedene Quellen zu differenzieren, da sich die PCE-C-Cl-Isotopensignaturen zwischen Standorten oft unterscheiden können. Wie die vorliegenden Resultate zeigen, ist jedoch in der Schweiz diese Methode für PCE nur beschränkt einsetzbar, da eine Unterscheidung der einzelnen Quellen nicht immer eindeutig ist. Auch zwischen zwei Standorten, bei denen CKW zu unterschiedlichen Zwecken zum Einsatz kamen, kann nicht zwingend erwartet werden, dass sich deren $\mathrm{C}$-Cl-Isotopensignatur unterscheidet. Da 
Tab. 2 C-Cl-Isotopensignatur von zehn PCE-belasteten Schweizer Standorten. Maximum C- und Cl-Isotopenverschiebung innerhalb jedes untersuchten Standortes (diese Studie) und innerhalb der vorher untersuchten Standorte, wo PCE reduktive Dechlorierung stattfindet (nach Wiegert et al. 2012; Badin et al. 2014). Die Standartabweichung entspricht der Standartabweichung der Messwerte aller Messstellen eines Standortes oder der analytischen Messunsicherheit, falls die letztere größer ist; a.U.: analytische Unsicherheit

\begin{tabular}{|c|c|c|c|c|}
\hline Standort & $\delta^{37} \mathrm{Cl}[\%$ ] & $\begin{array}{l}\delta^{37} \mathrm{Cl} \text { max. Unterschied zwischen } \\
\text { Min. und Max. }\end{array}$ & $\delta^{13} \mathrm{C}[\% 0]$ & $\begin{array}{l}\delta^{13} \mathrm{C} \text { max. Unterschied zwi- } \\
\text { schen Min. und Max. }\end{array}$ \\
\hline$\overline{\mathrm{SA}}$ & $0,1 \pm 0,1$ & 0,3 & $-25,1 \pm 0,5$ & 1,2 \\
\hline SB & $-0,5 \pm 0,2$ & 0,6 & $-24,4 \pm 0,4$ & 0,9 \\
\hline $\mathrm{SC}$ & $0,0 \pm 0,1$ & 0,2 & $-23,7 \pm 0,1$ & $0<$ a.U. \\
\hline SD & $0,6 \pm 0,2$ & 0,4 & $-26,0 \pm 0,6$ & 1,2 \\
\hline SE & $0,4 \pm 0,3$ & 0,6 & $-24,9 \pm 0,6$ & 1,3 \\
\hline $\mathrm{SF}$ & $0,0 \pm 0,2$ & $0<$ a.U. & $-25,7 \pm 0,3$ & 0,6 \\
\hline SG & $0,1 \pm 0,3$ & 0,6 & $-24,7 \pm 0,5$ & 0,9 \\
\hline $\mathrm{SH}$ & $0,1 \pm 0,4$ & 0,5 & $-25,8 \pm 0,1$ & 0,1 \\
\hline SI & $0,0 \pm 0,2$ & 0,3 & $-25,5 \pm 0,5$ & 1,2 \\
\hline SJ & $0,0 \pm 0,3$ & 0,5 & $-24,1 \pm 0,2$ & 0,4 \\
\hline X (Badin et al. 2014) & & 1,1 & & 0,8 \\
\hline Y (Badin et al. 2014) & & 0,7 & & 2,2 \\
\hline Transekt 1 (Wiegert et al. 2012) & & 1,4 & & 3,0 \\
\hline Transekt 2 (Wiegert et al., 2012) & & 1,9 & & 1,5 \\
\hline
\end{tabular}

die C-Cl-Isotopenanalyse in einigen Fällen jedoch erfolgreich zur Quellenidentifizierung angewandt werden konnte (Palau et al. 2014) und da gewisse Standorte dieser Studie unterschiedliche Isotopensignaturen aufweisen, kann man abschließend sagen, dass der Erfolg dieser Methode standortabhängig ist. Eine Vorstudie sollte also durchgeführt werden, um abzuklären, ob in einem bestimmten Fall unterschiedliche Quellen effektiv eine unterschiedliche Signatur aufweisen. Diese Schlussfolgerungen gelten dabei nur für PCE-Fälle, da keine anderen Schadstoffe betrachtet wurden.

Diese Methode ist damit als ein ergänzendes Werkzeug in Betracht zu ziehen, nachdem eine sorgfältige Standortuntersuchung durchgeführt worden ist oder wenn die Entnahme einiger zusätzlicher Proben zur Isotopenanalyse in Quellennähe ohne großen Mehraufwand möglich ist. Richtlinien zur Anwendung dieser Methode wurden bereits veröffentlicht (Hunkeler et al. 2008; Eisenmann \& Fischer 2010). Dabei wurden unterschiedliche Szenarien für die Anwendung der Methode unterschieden. Weil die CKW-Dechlorierung zu einer Änderung des Isotopenverhältnisses führt (Clark \& Fritz 1997; Hunkeler et al. 1999), ist es außerdem wichtig sicherzustellen, dass die Untersuchung der C-Cl-Isotopensignatur in der Nähe der Quelle durchgeführt wird, wo die geringste Dechlorierung, d. h. der geringste Abbau, zu erwarten ist. Wenn sich eine im Abstrombereich befindliche Belastung mittels C-Cl-Isotopenanalyse einer Quelle zuordnen lässt, muss die Dechlorierung berücksichtigt werden. Um sicherzustellen, dass das in Quellennähe gemessene Isotopenverhältnis dem initialen Isotopenverhältnis entspricht und sich mit der Zeit nicht ändert, sollten Proben zu zwei verschiedenen Zeitpunkten genommen und miteinander verglichen werden. Dies kann eine Fehlinterpretation der entsprechenden Resultate verhindern.

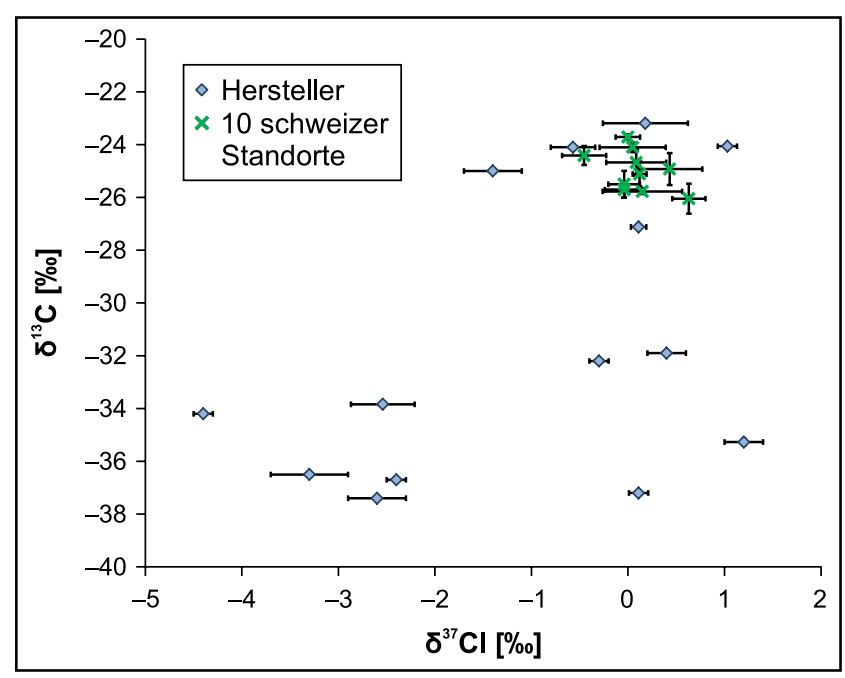

Abb. 2 Vergleich der PCE-C-Cl-Isotopensignatur zwischen den zehn Schweizer Standorten (diese Studie) und der Isotopensignatur von PCE von verschiedenen Herstellern (nach vanWarmerdam 1995; Jendrzejewski et al. 2001 und Mc Hugh et al. 2011). Die Standartabweichung entspricht der Standartabweichung der Messwerte aller Messstellen eines Standortes oder der analytischen Messunsicherheit, falls die letztere größer ist

Danksagung Diese Studie wurde im Rahmen des Marie Curie Initial Training Network ,ADVOCATE - Advancing sustainable in situ remediation for contaminated land and groundwater" durchgeführt, und von der Europäische Kommission, Marie Curie Actions Project No. 265063 finanziert. Den Standorteigentümern, die die Probennahmen ermöglicht haben, den Mitarbeitern von Ingenieurbüros sowie den Kollegen von Ämtern, die die Daten von den Standorten zur Verfügung gestellt und den Zustand der Standorte mit Geduld erklärt haben, wird herzlichst gedankt. Weiter gebührt ein herzlicher Dank Jordi Palau und Dimitri Boulaz für ihre wertvolle Hilfe während der Feldarbeit und Daniel Bouchard, Jordi Palau und Simon Jeannottat für Ihre Hilfe bei den Isotopenmessungen. Weiter wird Vivien Hakoun und Radu Slobodeanu für die konstruktiven Diskussionen bezüglich der Datenauswertung gedankt. 
Abb. 3 Isotopensignatur von PCE an zehn Schweizer Standorten. Die Standartabweichung entspricht der Standartabweichung der Messwerte aller Messstellen eines Standortes oder der analytischen Messunsicherheit, falls die letztere größer ist

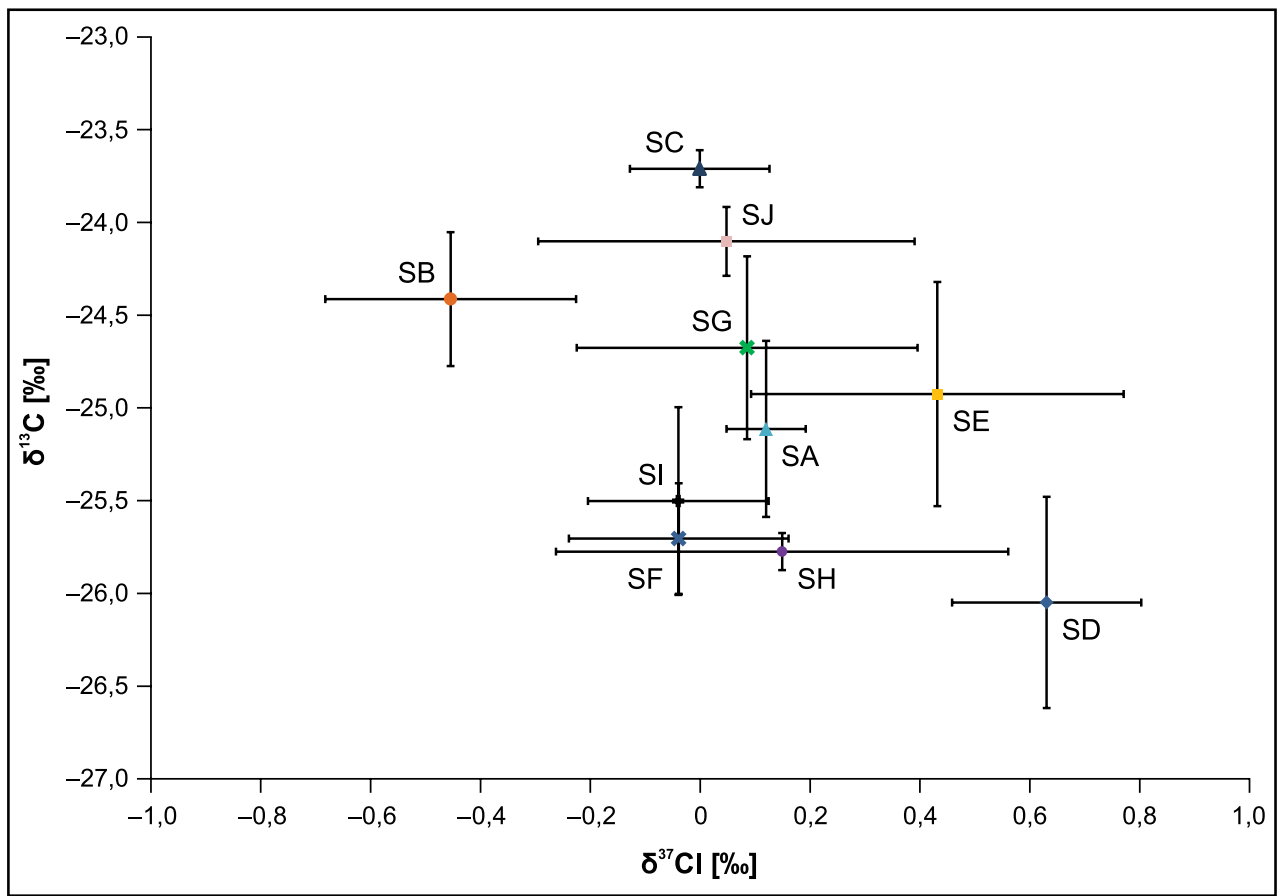

\section{Literatur}

Aeppli, C., Holmstrand, H., Andersson, P., Gustafsson, O.: Direct compound-specific stable chlorine isotope analysis of organic compounds with quadrupole GC/MS Using standard isotope bracketing. Anal. Chem. 82(1), 420-426 (2010)

Amaral, H.I., Aeppli, C., Kipfer, R., Berg, M.: Assessing the transformation of chlorinated ethenes in aquifers with limited potential for natural attenuation: added values of compound-specific carbon isotope analysis and groundwater dating. Chemosphere. 85(5), 774-781 (2011)

Badin, A., Buttet, G., Maillard, J., Holliger, C., Hunkeler, D.: Multiple dual $\mathrm{C}-\mathrm{Cl}$ isotope patterns associated with reductive dechlorination of tetrachloroethene. Environ. Sci. Technol. 48(16), 9179-9186 (2014)

Bernstein, A., Shouakar-Stash, O., Ebert, K., Laskov, C., Hunkeler, D., Jeannottat, S., Sakaguchi-Söder, K., Laaks, J., Jochmann, M.A., Cretnik, S., Jager, J., Haderlein, S.B., Schmidt, T.C., Aravena, R., Elsner, M.: Compound-specific chlorine isotope analysis: a comparison of gas chromatography/isotope ratio mass spectrometry and gas chromatography/quadrupole mass spectrometry methods in an interlaboratory study. Anal. Chem. 83(20), 7624-7634 (2011)

BIPM, I., IFCC, ISO, IUPAC, IUPAP, OIML: Guide to the expression of uncertainty in measurement. International Organization for Standardization, Geneva, (1993)

Blessing, M., Schmidt, T.C., Dinkel, R., Haderlein, S.B.: Delineation of multiple chlorinated ethene sources in an industrialized area a forensic field study using compound-specific isotope analysis. Environ. Sci. Technol. 43(8), 2701-2707 (2009)

Bloom, Y., Aravena, R., Hunkeler, D., Edwards, E., Frape, S.K.: Carbon isotope fractionation during microbial dechlorination of trichloroethene, cis -1,2-dichloroethene, and vinyl chloride: Implications for assessment of natural attenuation. Environ. Sci. Technol. 34(13), 2768-2772 (2000)

Clark, I.D., Fritz, P.: Environmental isotopes in hydrogeology, S 328. Lewis Publishers, Boca Raton (1997)
Doherty, R.E.: A history of the production and use of carbon tetrachloride, tetrachloroethylene, trichloroethylene and 1,1,1-trichloroethane in the United States: Part 1- Historical background; Carbon tetrachloride and tetrachloroethylene. Environ. Forens. 1(2), 69-81 (2000)

Eberts, S.M., Braun, C., Jones, S.: Compound-specific isotope analysis: questioning the origins of a trichloroethene plume. Environ. Forens. 9(1), 85-95 (2008)

Eisenmann, H., Fischer, A.: Isotopenuntersuchungen in der Altlastenbewertung. In: Franzius, V., Altenbockum, M., Gerhold, T. (Hrsg.) Handbuch der Altlastensanierung und Flächenmanagement, S 47. Hüthig Jehle Rehm, München (2010)

Hitzfeld, K.L., Gehre, M., Richnow, H.-H.: A novel online approach to the determination of isotopic ratios for organically bound chlorine, bromine and sulphur. Rapid. Commun. Mass. Spectrom. 25(20), 3114-3122 (2011)

Holt, B.D., Sturchio, N.C., Abrajano, T.A., Heraty L.J.: Conversion of chlorinated volatile organic compounds to carbon dioxide and methyl chloride for isotopic analysis of carbon and chlorine. Anal. Chem. 69(14), 2727-2733 (1997)

Hunkeler, D., Aravena, R., Butler, B.J.: Monitoring microbial dechlorination of tetrachloroethene (PCE) in groundwater using compound-specific stable carbon isotope ratios: Microcosm and field studies. Environ. Sci. Technol. 33(16), 2733-2738 (1999)

Hunkeler, D., Chollet, N., Pittet, X., Aravena, R., Cherry, J.A., Parker, B.L.: Effect of source variability and transport processes on carbon isotope ratios of TCE and PCE in two sandy aquifers. J. Contam. Hydrol. 74(1-4), 265-282 (2004)

Hunkeler, D., Meckenstock, R.U., Lollar Sherwood, B., Schmidt, T.C., Wilson, J.T.: A Guide for Assessing Biodegradation and Source Identification of Organic Groundwater Contaminants Using Compound Specific Isotope Analysis (CSIA), Environmental Protection Agency. EPA/600/R -08/148 (2008)

Jeannottat, S., Hunkeler, D.: Chlorine and carbon isotopes fractionation during volatilization and diffusive transport of trichloroethene in the unsaturated zone. Environ. Sci. Technol. 46(6), 3169-3176 (2012) 
Jeannottat, S., Hunkeler, D.: Can soil gas VOCs be related to groundwater plumes based on their isotope signature? Environ. Sci. Technol. 47(21), 12115-12122 (2013)

Jendrzejewski, N., Eggenkamp, H.G.M., Coleman, M.L.: Characterisation of chlorinated hydrocarbons from chlorine and carbon isotopic compositions: scope of application to environmental problems. Appl. Geochem. 16(9-10), 1021-1031 (2001)

Jin, B.A., Laskov, C., Rolle, M., Haderlein, S.B.: Chlorine isotope analysis of organic contaminants using GC-qMS: method optimization and comparison of different evaluation schemes. Environ. Sci. Technol. 45(12), 5279-5286 (2011)

Kaown, D., Shouakar-Stash, O., Yang, J., Hyun, Y., Lee, K.-K.: Identification of multiple sources of groundwater contamination by dual isotopes. Ground. Water. 52(6), 875-885 (2014)

Lojkasek-Lima, P., Aravena R., Parker, B.L., Cherry, J.A.: Fingerprinting TCE in a bedrock aquifer using compound-specific isotope analysis. Ground. Water. 50(5), 754-764 (2012)

Lollar, B.S., Slater, G.F., Ahad, J., Sleep, B., Spivack, J., Brennan, M., MacKenzie, P.: Contrasting carbon isotope fractionation during biodegradation of trichloroethylene and toluene: Implications for intrinsic bioremediation. Org. Geochem. 30(8A), 813-820 (1999)

McHugh, T., Kuder, T., Fiorenza, S., Gorder, K., Dettenmaier, E., Philp, P.: Application of CSIA to distinguish between vapor intrusion and indoor sources of VOCs. Environ. Sci. Technol. 45(14), 5952-5958 (2011)

Moran, M.J., Zogorski, J.S., Squillace, P.J.: Chlorinated solvents in groundwater of the United States. Environ. Sci. Technol. 41(1), 74-81 (2007)
Nijenhuis, I., Schmidt, M., Pellegatti, E., Paramatti, E., Richnow, H.H., Gargini, A.: A stable isotope approach for source apportionment of chlorinated ethene plumes at a complex multi-contamination events urban site. J. Contam. Hydrol. 153, 92-105 (2013)

Palau, J., Marchesi, M., Chambon, J.C.C., Aravena, R., Canals, À., Binning, P.J., Bjerg, P.L., Otero, N., Soler, A.: Multi-isotope (carbon and chlorine) analysis for fingerprinting and site characterization at a fractured bedrock aquifer contaminated by chlorinated ethenes. Sci. Total Environ. 475, 61-70 (2014)

Pankow, J. F., Cherry, J.A.: Dense chlorinated solvents and other DNAPLs in groundwater: history, behavior, and remediation. Waterloo Press, Portland (1996)

Sakaguchi-Söder, K., Jager, J., Grund, H., Matthäus, F., Schüth, C.: Monitoring and evaluation of dechlorination processes using compound-specific chlorine isotope analysis. Rapid. Commun. Mass. Spectrom. 21(18), 3077-3084 (2007)

Slater, G.F., Dempster, H.S., Lollar, B.S., Ahad, J.: Headspace analysis: a new application for isotopic characterization of dissolved organic contaminants. Environ. Sci. Technol. 33(1), 190-194 (1999)

vanWarmerdam, E.M., Frape, S.K., Aravena, R., Drimmie, R.J., Flatt, H., Cherry, J.A.: Stable chlorine and carbon isotope measurements of selected chlorinated organic solvents. Appl. Geochem. 10(5), 547-552 (1995)

Wiegert, C., Aeppli, C., Knowles, T., Holmstrand, H., Evershed, R., Pancost, R.D., Macháčková, J., Gustafsson, Ö.: Dual carbon-chlorine stable isotope investigation of sources and fate of chlorinated ethenes in contaminated groundwater. Environ. Sci. Technol. 46(20), 10918-10925 (2012) 